\title{
Armin Glatzmeier. 2019. Gerichte als politische Akteure. Zur funktionalen Rolle der Verfassungsgerichtsbarkeit in Demokratien.
}

\author{
Baden-Baden: Nomos. 555 Seiten, 119€, ISBN 1868-1808
}

\section{Werner Reutter}

Online publiziert: 9. Juni 2020

(C) Der/die Autor(en) 2020

Am 27. April 2020 hat der Bayerische Verwaltungsgerichtshof $\S 2$ Abs. 4 und 5 der 2. Bayerischen Infektionsschutzmaßnahmeverordnung für unvereinbar erklärt mit Art. 3 Abs. 1 GG. Die in der Verordnung vorgesehene Möglichkeit, die Öffnung von Einzelhandelsgeschäften während der aktuellen Corona-Pandemie nur bis zu einer Größe von 800 qm Verkaufsfläche zu erlauben, verstoße ,,aller Voraussicht nach gegen den Gleichheitsgrundsatz“, weil damit ,im Wesentlichen ungleiche Sachverhalte“ gleichbehandelt würden und zwar ohne „sachliche Rechtfertigung" (VGH München, Beschluss v. 27.04.2020 - 20 NE 20.793). Armin Glatzmeier wird dieser Beschluss gefallen. Denn er belegt eine wesentliche These seiner Studie. Die Überprüfung rechtlicher Normen auf ihre Vereinbarkeit mit der Verfassung finde, so Glatzmeier, eben nicht nur in Verfassungsgerichten statt, sondern könne auch durch Fachgerichte erfolgen. Die ,,verfassungsrechtliche Qualität eines Rechtsstreits“ ergibt sich mithin aus dem ,Verfahrensgegenstand“ (S. 512) und nicht notwendig aus der Institution, die darüber entscheidet. Damit einher geht, dass Glatzmeier der Trennung von Recht und Politik, wie sie insbesondere in der deutschen Staatsrechtslehre gepflegt wird, nichts abgewinnen kann. Im Gegenteil, Glatzmeier folgt der amerikanischen Sicht und betrachtet das Gerichtssystem als eine „branch of government“, das gesetztes Recht nicht nur anwendet, sondern selbst schöpft. Es sei, wie es der U.S. Supreme Court 1803 in seiner bahnbrechenden Entscheidung Marbury vs. Madison postuliert hat, ,emphatically the province and duty of the judicial department to say what the law is“" (Marbury v. Madison, 5. U.S. (1 Cr.) 137, 177 (1803)).

Bei Armin Glatzmeiers Studie handelt es sich um die überarbeitete und leicht aktualisierte Fassung seiner 2016 von der Philosophischen Fakultät der Universi-

PD Dr. W. Reutter $(\bowtie)$

Institut für Sozialwissenschaften, Humboldt-Universität zu Berlin, Unter den Linden 6, 10099 Berlin, Deutschland

E-Mail: werner.reutter@rz.hu-berlin.de 
tät Passau angenommenen Dissertation. Das mag erklären, warum Glatzmeier unbeschadet eines knapp 30 Seiten umfassenden Literaturverzeichnisses - einen „Mangel an sozial- und geisteswissenschaftlicher Literatur zur Verfassungsgerichtsbarkeit" (S. 51) diagnostiziert. Seine Analyse ist - wie viele Studien in diesem Forschungsfeld - mit über 550 Seiten recht voluminös und folgt keinem alle Kapitel umfassenden Forschungsdesign. Vielmehr verwendet er einen „Methodenmix“. Je nach Teilfrage stützt er sich auf divergierende Fälle und variierende Analyseverfahren. Glatzmeier bezeichnet dieses Vorgehen als ,erklärende[s] Verstehen“ (S. 46). Im historischen Teil bezieht sich Glatzmeier auf die USA und die BRD sowie am Rande - auf Österreich und Großbritannien. Ansonsten fußt die Untersuchung auf die Verfassungen aller 28 EU-Staaten sowie der USA, sodass sich letztlich nur ein Kriterium erkennen lässt, mit dem Glatzmeier seine „Länderauswahl“ (S. 47) begründet. Es sind alles Demokratien. Diese Demokratien sind alt und jung, haben ein präsidentielles oder parlamentarisches Regierungssystem, sind Bundes- oder Einheitsstaat, besitzen eine zentrale oder dezentrale Verfassungsgerichtsbarkeit und folgen dem Common Law oder dem Civil Law (S. 47).

Glatzmeiers Anspruch ist groß. Er möchte einen „Beitrag zur Vereinheitlichung“ der - doch recht heterogenen - Forschung auf diesem Gebiet leisten und den ,funktionalen Systembeitrag der Verfassungsgerichtsbarkeit (auch komparativ) angemessen“ erfassen (S. 27). Mit seiner Arbeit stellt Glatzmeier die Frage „,nach der Rolle von Verfassungsgerichten bzw. Verfassungsgerichtsbarkeit im Kontext des politischen Systems“ (S. 509). Wie erwähnt, ist dabei unterstellt, dass sich Funktion und Institution trennen lassen, ja sogar getrennt werden müssen. Verfassungsrechtsprechung ist Aufgabe des gesamten Gerichtssystems eines Landes, und zwar auch dann, wenn dieses Land dem österreichischen Beispiel folgt und einen Gerichtshof geschaffen hat, das über die ausschließliche Kompetenz zum judicial review verfügt. Gleichzeitig will Glatzmeier mit dieser theoretischen Grundierung die Diskussion um die Rolle von Gerichten in politischen Systemen normativ immunisieren. Er hält die Trennung von Recht und Politik - ganz in systemtheoretischer Manier - ohnehin für künstlich und im Grunde Ausfluss eines überholten deutschen Sonderweges in der Forschung zur Verfassungsgerichtsbarkeit. Gerichte und damit auch Verfassungsgerichte nehmen eine ,aktive, politikgestaltende Rolle“ ein (S. 44). Das liegt in der „Natur der Sache“, wie Glatzmeier im Anschluss an Dieter Grimm (2011) feststellt (S. 43). Denn Gerichte treffen zwangsläufig „Policy-Entscheidungen und nehmen Einfluss auf den Politikprozess“ (S. 43). Das ist schwer zu bestreiten. Die Frage ist nur, wie sich solche Befunde theoretisch einordnen und normativ begründen lassen. Dieser Frage geht Glatzmeier in drei großen Schritten nach: historisch, methodenkritisch und systematisch.

Seine historische Analyse konzentriert sich auf die klassischen Fälle: die USA und die BRD. Außerdem erwähnt er in diesem Zusammenhang noch Österreich und - Überraschung! - Großbritannien. Mit der Rekonstruktion der Entstehung von Verfassungsgerichtsbarkeit will Glatzmeier vor allem herausarbeiten, ,welche konkreten Funktionen“ den Verfassungsgerichten bei ihrer Entstehung zugeschrieben wurden oder ob es sich eher um einen langfristigen Anpassungsprozess handelt, in dem die zu erbringenden Systemfunktionen der Institution zugewachsen sind. Obwohl sich zwischen der amerikanischen und deutschen Herausbildung des richterlichen Prü- 
fungsrechtes gewisse Parallelen finden lassen, stechen doch die unterschiedlichen Motive hervor, die die Entstehungsprozesse prägten: Während im deutschen Fall der Schutz der Verfassung und der Grundrechte im Vordergrund stand, lag in der amerikanischen Verfassungsdiskussion der Schwerpunkt auf der „Absicherung der Bundeskompetenzen im föderalen Staat" (S. 106).

Im methodenkritischen Teil (S. 107-283) will Glatzmeier zum einen „Klarheit bezüglich des Forschungsgegenstandes“" schaffen sowie „Probleme bei der vergleichenden Erforschung von Verfassungsgerichten“ aufzeigen (S. 48). In diesem Zusammenhang rekonstruiert Glatzmeier wesentliche Ansätze aus der vergleichenden Forschung (u. a. von Cappelletti, Tsebelis, Kneip, Lijphart, Tushnet) und kommt zu dem doch recht ernüchternden - aber auch wenig überraschenden - Fazit, dass sich weder von einer einheitlichen Begrifflichkeit noch von einer methodischen Kohärenz in der vergleichenden Forschung zur Verfassungsgerichtsbarkeit sprechen ließe. Denn nach Glatzmeier konkurrierten ,quantitative und qualitative Ansätze“ (S. 282), meine der Begriff der Verfassungsrechtsprechung bei den einzelnen Autoren Unterschiedliches und werde die Stärke von Verfassungsgerichten variierend erschlossen. Insgesamt erkennt Glatzmeier „Zwei akute Probleme bei der vergleichenden Forschung von Verfassungsgerichten" (S. 285): Zum einen sei das Forschungsfeld „begrifflich unklar strukturiert“; zum anderen werde die „Stärke“ von Verfassungsgerichten unterschiedlich bestimmt (S. 285).

Im anschließenden Hauptteil seiner Untersuchung (S. 285-507) adressiert Glatzmeier diese beiden Probleme: Er nimmt dafür noch einmal eine Begriffsklärung vor und plädiert erneut dafür, in der Forschung zwischen Verfassungsgericht und Verfassungsgerichtsbarkeit zu trennen. Auf dieser Grundlage und im Anschluss an die Systemtheorie von Almond und Powell (1966 sowie Almond et al. 2010) entwickelt Glatzmeier ein Modell verfassungsgerichtlicher Kontrolle, dessen Erklärungspotential er an einem Fallbeispiel illustriert. Hier arbeitet er den Beitrag heraus, den Gerichte bei der rechtlichen Anerkennung von Transpersonen in Deutschland, Großbritannien und den USA geleistet haben. Er verwendet in diesem Teil ausschließlich qualitative Methoden und untersucht anhand ausgewählter Urteile, ob und inwiefern Gerichte in den drei erwähnten Ländern an der Entwicklung in diesem Politikfeld beteiligt waren und zwar in allen Phasen eines policy-cycle (S. 420-514). Daraus entwickelt Glatzmeier schließlich das Modell eines verfassungsgerichtlichen Kontrollregimes.

Zweifellos ist Glatzmeier mit seiner Studie ein großer Wurf gelungen. Die erwähnte Fallstudie ist ebenso überzeugend wie die vorgeschlagene Trennung von Institution und Funktion. Auch die Herausarbeitung der unterschiedlichen Funktionen, die Gerichte für politische Systeme erbringen können, gelingt ihm überzeugend. Das ist sicher nicht wenig. Allerdings hätte der Wurf weiter ausfallen können. Weniger wäre an manchen Stellen mehr gewesen. So fällt etwa der methodenkritische zweite Teil zu umfangreich aus und wird auch den Leistungen anderer Forscher nicht immer gerecht. Ebenso hat sich dem Autor dieser Zeilen nicht erschlossen, warum noch einmal die Entstehung der Verfassungsgerichtsbarkeit in den USA und der BRD darzustellen war. Glatzmeier fügt dem Forschungsstand hier nichts Neues bei. Überrascht hat den Rezensenten auch, dass einflussreiche Arbeiten unberücksichtigt blieben (Pócza et al. 2017; Hirschl 2007). Nicht überzeugen kann 
der Versuch Glatzmeiers, die Rolle von Verfassungsgerichtsbarkeit normativ zu immunisieren. Die aktuellen Entwicklungen in Ungarn oder Polen verweisen darauf, dass sich Demokratie und Verfassungsrechtsprechung in einem Spannungsverhältnis befinden (und befinden müssen), das sich mit dem ausgefeilten Instrumentarium, das Glatzmeier bereitstellt, zwar virtuos erschließen und beschreiben lässt, aber als wertneutrale Systemfunktion schwerlich adäquat erfasst werden kann.

Funding Open Access funding provided by Projekt DEAL.

Open Access Dieser Artikel wird unter der Creative Commons Namensnennung 4.0 International Lizenz veröffentlicht, welche die Nutzung, Vervielfältigung, Bearbeitung, Verbreitung und Wiedergabe in jeglichem Medium und Format erlaubt, sofern Sie den/die ursprünglichen Autor(en) und die Quelle ordnungsgemäß nennen, einen Link zur Creative Commons Lizenz beifügen und angeben, ob Änderungen vorgenommen wurden.

Die in diesem Artikel enthaltenen Bilder und sonstiges Drittmaterial unterliegen ebenfalls der genannten Creative Commons Lizenz, sofern sich aus der Abbildungslegende nichts anderes ergibt. Sofern das betreffende Material nicht unter der genannten Creative Commons Lizenz steht und die betreffende Handlung nicht nach gesetzlichen Vorschriften erlaubt ist, ist für die oben aufgeführten Weiterverwendungen des Materials die Einwilligung des jeweiligen Rechteinhabers einzuholen.

Weitere Details zur Lizenz entnehmen Sie bitte der Lizenzinformation auf http://creativecommons.org/ licenses/by/4.0/deed.de.

\section{Literatur}

Almond, Gabriel A., und G. Bingham Powell. 1966. Comparative politics today. A world view. Boston: Little, Brown and Co.

Almond, Gabriel A., G. Bingham Powell, Russell J. Dalton, und Kaare Strøm. 2010. Comparative politics today. A world view, 9. Aufl., New York: Pearson Longman.

Grimm, Dieter. 2011. „Was das Grundgesetz will, ist eine politische Frage,“ Frankfurter Allgemeine Zeitung, 22. Dezember 2011. https://www.faz.net/aktuell/feuilleton/bundesverfassungsgericht-wasdas-grundgesetz-will-ist-eine-politische-frage-11575149.html?printPagedArticle=true\#pageIndex _ 2. Zugegriffen: 15. April 2020.

Hirschl, Ran. 2007. Towards Juristocracy. The origins and consequences of the new constitutionalism. Cambridge: Harvard University Press.

Pócza, Kálmán, Gábor Dobos, und Attila Gyulai. 2017. How to measure the strength of judicial decisions: a methodological framework. German Law Journal 18(6):1557-1586. 